\section{P04.25 SEXUAL HEALTH PROBLEMS AMONG ADULT MALES OF NORTH INDIA: A COMMUNITY BASED STUDY}

Misra Puneet*, Kant Shashi, Rai Sanjay, Kumar Arvind. Centre for Community Medicine, All India Institute of Medical Science, New Delhi, India

10.1136/sextrans-2015-052270.279

Introduction Sexual health problem, though less studied, is an important public health problem. The magnitude of sexual health problems in Indian population, due to various myths and cultural taboos, may be alarming. However, there has never been a community based study to document the magnitude of sexual health problems.

Objective To estimate the prevalence of different types of sexual health problems in adult males aged 18 or more in rural Haryana and identify various factors associated with reported sexual health problems.

Methodology The study was conducted in a sample of 900 adult males of Faridabad district of Haryana. Socio-demographic characteristics, lifestyle practices, sexual habits and practices and selfreported sexual problems were captured. Sexual health problems studied were erectile dysfunction (ED), premature ejaculation (PME), nocturnal emission, loss of libido, defect in semen and genitalia and culture bound sexual neurosis. Bi-variate analysis was done to find out the factors associated with presence of sexual health problems.

Results Nearly $50 \%$ of the participants reported having any of the sexual health problems. Common sexual health problems reported were ED (20\%), PME (14.6\%), nocturnal emission $(13.6 \%)$, defect in semen (33\%), loss of libido (20\%), and culture bound sexual neurosis (5\%). Adults males of more than 30 years of age and who were current alcoholic and smoker with duration of intake more than 10 years, cannabis users, having history of snoring and chronic illness were at significantly higher risk of having a sexual health problem.

Conclusion The prevalence of sexual health problems was high in the study population. High prevalence of sexual health problems warrants a community level intervention as most of the factors associated were modifiable. A holistic care and support mechanism to deal with sexual health problems is required.

\section{P04.26 ROBUSTNESS OF CAPTURING BEHAVIOURAL AND SEXUAL LIFESTYLE DATA FOR A COMPLEX CLINICAL STUDY USING INTERNET-BASED COMPUTER ASSISTED SELF-INTERVIEW}

${ }^{1}$ AV Nori* ${ }^{*}{ }^{1,2} \mathrm{PE}$ Hay, ${ }^{1} \mathrm{PD}$ Butcher, ${ }^{1,2} \mathrm{ST}$ Sadiq. ${ }^{1}$ Institute for Infection and Immunity, St George's University of London, London, UK; ${ }^{2}$ Department of Genitourinary and Medicine, St George's Healthcare NHS Trust, London, UK

\subsection{6/sextrans-2015-052270.280}

Introduction Sexual behaviour and vaginal practices impact on vaginal pathology, but accurately capturing such data for multisite clinical studies is logistically difficult. Computer assisted selfinterviews (CASI), are useful for capturing sensitive information for community surveys but require programming expertise or expensive software. We piloted an open-source software designed internet-based CASI, using previously validated questions for a multi-site clinical study on the vaginal microbiome. Methods The CASI question-structure was constructed from the third UK National Survey of Sexual Attitudes and Lifestyles using LimeSurvey open source software and consisted of 71 questions, delivered through a responsive algorithm, covering demographics, sexual behaviour, vaginal practices, contraception and life-time smoking history. Patients could decline questions and the CASI was delivered to participants online. Bacterial vaginosis (BV) status was determined by Nugent scores from Gramstained lateral vaginal swab smears.

Results Data from 155 women with available Nugent scores were included. Mean time to completing CASI was $303 \mathrm{~s}$ (Range: $111.76-670.15$ s 95\% CI \pm 16.83 s). There were no missing data. The question most participants declined to answer was "number of one-off partners" (14 participants, 9\%). White ethnicity and oestrogen-based contraceptives were inversely related with BV (Odds ratios (OR): 0.33 [95\% CI: 0.16-0.67] and 0.41 [0.17-0.97] respectively) and lifetime smoking of $>1$ pack-years and regular vaginal douching were directly related with BV (OR: 2.56 [1.21-5.41] and 2.03 [1.01-4.09] respectively). Nearly $50 \%$ of women reported daily vaginal douching and $>90 \%$ reported using feminine care products in the preceding month.

Conclusion The CASI delivered a complete dataset, the findings from which were consistent with published associations of BV, demonstrating robustness. Web-based CASI is an efficient method of collecting sensitive sexual and behavioural data within a complex clinical study, from patients recruited in busy clinical settings, and can be developed using open-source questionnaire software without the need for coding expertise.

Disclosure of interest statement This work was done as part of the Electronic Self-Testing Instruments for Sexually Transmitted Infection (eSTI2) Consortium funded under the UKCRC Translational Infection Research (TIR) Initiative supported by the Medical Research Council (Grant Number G0901608). The funding sources had no involvement in the study design or conduct; the collection, analysis and interpretation of data; the preparation, review or approval of the manuscript; or the decision to submit the manuscript for publication. The views expressed are those of the authors and not necessarily those of the NHS, the MRC, or St George's, University of London. The authors declare no competing interests.

\section{P04.27 PARTNER-LEVEL ASSOCIATED FACTORS FOR INSERTIVE AND RECEPTIVE CONDOMLESS ANAL INTERCOURSE AMONG TRANSGENDER WOMEN IN LIMA, PERU}

${ }^{1}$ MF Satcher*, ${ }^{2}$ ER Segura, ${ }^{3}$ A Silva-Santisteban, ${ }^{4}$ S Sanchez, ${ }^{4} J R$ Lama, ${ }^{2} J L$ Clark. ${ }^{1}$ South American Program in HIV Prevention Research (SAPHIR), David Geffen School of Medicine, University of California, Los Angeles, CA, USA; ${ }^{2}$ Program in Global Health, Division of Infectious Diseases, Department of Medicine, David Geffen School of Medicine, University of California, Los Angeles, CA, USA; ${ }^{3}$ Unit of Health, Sexuality and Human Development, Cayetano Heredia University School of Public Health, Lima, Peru; ${ }^{4}$ Asociación Civil Impacta Salud Y Educación, Lima, Peru

\subsection{6/sextrans-2015-052270.281}

Introduction Partner type has been associated with condomless anal intercourse (CAI) among TW in Peru. We characterised the relationship between partnership characteristics and receptive (RCAI) versus insertive CAI (I-CAI) among TW.

Methods We analysed cross-sectional, egocentric data from TW screened for a 2012-2014 Partner Management study in Lima, Peru. We included self-identified TW reporting anal intercourse (AI) with at least 1 of their last 3 non-female partners. Generalised estimating equations with Poisson distribution were used to assess prevalence ratios (PR) with 95\% CIs for R-CAI and I-CAI during the last sexual encounter by partner type (adjusted for 